\title{
A Continuity Property of the Entropy Density for Spin Lattice Systems
}

\author{
M. Fannes ${ }^{\star}$ \\ Universiteit Leuven, Belgium \\ Received December 15, 1972
}

\begin{abstract}
The entropy density of spin lattice systems is known to be a weak ${ }^{\star}$ upper semi-continuous functional on the set of the lattice invariant states. (It is even weak ${ }^{\star}$ discontinuous.) However we prove here that it is continuous with respect to the norm topology on those states.
\end{abstract}

\section{Preliminaries}

We consider a lattice $\mathbb{Z}^{d}$ of $N$ spin states per lattice site. By $\Lambda \subset \mathbb{Z}^{d}$ we will always mean a non-empty finite volume and by $V(\Lambda)$ the number of points in it.

To $A \subset \mathbb{Z}^{d}$ we associate the local algebra $\mathscr{A}_{A}$ of observables:

$$
\mathscr{A}_{\Lambda}=\mathscr{B}\left(\mathscr{H}_{\Lambda}\right) \quad \text { where } \quad \mathscr{H}_{\Lambda}=\bigotimes_{i \in \Lambda} \mathscr{H}_{i} \text { and each } \mathscr{H}_{i}
$$

is an isomorphic copy of the $N$-dimensional Hilbert space $\mathbb{C}^{N}$. For $\Lambda_{1} \subset \Lambda_{2}$ we trivially get an isometric embedding of $\mathscr{A}_{A_{1}}$ in $\mathscr{A}_{\Lambda_{2}}$ which maps $A$ into $A \otimes 1_{A_{2} \backslash A_{1}}$. This allows us to construct the $C^{*}$-algebra $\mathscr{A}$ of quasilocal observables:

$$
\mathscr{A}={\bigcup_{\Lambda \subset \mathbb{Z}^{d}} \mathscr{A}_{\Lambda}}^{n}
$$

The natural translation mappings $\tau_{x}: \mathscr{A}_{A} \rightarrow \mathscr{A}_{A+x}, x \in \mathbb{Z}^{\mathrm{d}}$ extend to a group $\left\{\tau_{x} \mid x \in \mathbb{Z}^{d}\right\}$ of automorphisms of $\mathscr{A}$. A state $\omega$ on $\mathscr{A}$ is called lattice invariant if $\omega \circ \tau_{x}=\omega$ for all $x \in \mathbb{Z}^{d}$. Let $\mathscr{E}$ denote the set of all lattice invariant states on $\mathscr{A}$.

For each state $\omega$ on $\mathscr{A}$ and for each $\Lambda \subset \mathbb{Z}^{d}$ there exists a unique density matrix $\varrho_{A} \in \mathscr{B}\left(\mathscr{H}_{A}\right)$ such that $\forall A \in \mathscr{A}_{A} \omega(A)=\operatorname{Tr} \varrho_{A} A$.

The local entropy density of the state $\omega$ is given by

$$
s_{\Lambda}(\omega)=-\frac{1}{V(\Lambda)} \operatorname{Tr} \varrho_{\Lambda} \log \varrho_{\Lambda} .
$$

* Aspirant van het Belgisch N.F.W.O. 
The existence of $s(\omega)=\lim _{\Lambda \rightarrow \infty} s_{\Lambda}(\omega)$ has been proved for $\omega \in \mathscr{E}$ in the sense of growing cubes [1].

The continuity properties of the map $\omega \rightarrow s(\omega)$ are important with respect to the variational formulation of statistical mechanics [1] and explicit calculations of the entropy density [2]. It has been proved that the map is upper semi-continuous for the weak* topology on $\mathscr{E}[1]$; however it can easily be proved that it is not weak* continuous [2]. Here we prove the norm continuity.

\section{A Continuity Property of the Entropy Density}

Lemma 1 (Lidskii [3]). Let $A, B$ be selfadjoint operators on the $n$-dimensional Hilbert space $H$. Denote by $\alpha_{k}, \beta_{k}$ and $\gamma_{k}, k=1, \ldots, n$ the repeated eigenvalues of $A, B$ and $A-B$ in ascending order. Then there exist numbers $\sigma_{k j}, \quad k, j=1, \ldots, n$ such that:

1) $\sigma_{k j} \geqq 0$.

2) $\sum_{k} \sigma_{k j}=\sum_{j} \sigma_{k j}=1$.

3) $\alpha_{k}-\beta_{k}=\sum_{j} \sigma_{k j} \gamma_{j}$.

Lemma 2. The function $f:[0,1] \rightarrow[0,1 / e]: x \rightarrow f(x)=-x \log x$ satisfies the inequality:

$$
|f(x)-f(y)| \leqq 2|y-x|+f(|y-x|) .
$$

Proof. For $0 \leqq x \leqq y \leqq 1$ we have:

$$
\begin{aligned}
\mid f(x) & -f(y) \mid \\
& =|-x(\log x-1)+y(\log y-1)+y-x| \\
& \leqq|-x(\log x-1)+y(\log y-1)|+y-x \\
& =\left|\int_{x}^{y} d t \log t\right|+y-x \\
& =-\int_{x}^{x+(y-x)} d t \log t+y-x \\
& \leqq-\int_{0}^{y-x} d t \log t+y-x=2(y-x)+f(y-x) .
\end{aligned}
$$

Theorem. The function $s: \mathscr{E} \rightarrow[0, \log N]: \omega \rightarrow s(\omega)$ is continuous with respect to the norm topology on $\mathscr{E}$.

Proof. Let $\omega_{1}, \omega_{2} \in \mathscr{E}$ and $\varrho_{\Lambda}^{1}, \varrho_{\Lambda}^{2}, \Lambda \subset \mathbb{Z}^{d}$ the corresponding density matrices. 
Denote by $\lambda_{k}^{1}, \lambda_{k}^{2}, \mu_{k}, k=1, \ldots, N^{V(\Lambda)}$ the repeated eigenvalues of $\varrho_{A}^{1}, \varrho_{A}^{2}$ and $\varrho_{A}^{1}-\varrho_{A}^{2}$ in ascending order. By Lemma 1

$$
\begin{aligned}
\sum_{k}\left|\lambda_{k}^{1}-\lambda_{k}^{2}\right|=\sum_{k}\left|\sum_{j} \sigma_{k j} \mu_{j}\right| \leqq \sum_{k} \sum_{j} \sigma_{k j}\left|\mu_{j}\right| \\
=\sum_{j}\left|\mu_{j}\right|=\operatorname{Tr}\left|\varrho_{A}^{1}-\varrho_{A}^{2}\right|=\sup _{\substack{\|A\| \leq 1 \\
A \in \mathscr{\mathscr { A }} A}}\left|\operatorname{Tr}\left(\varrho_{A}^{1}-\varrho_{A}^{2}\right) A\right| \\
=\sup _{\substack{\|A\| \leq 1 \\
A \in \mathscr{A} A}}\left|\left(\omega_{1}-\omega_{2}\right)(A)\right| \leqq \sup _{\substack{\|A\| \leqq 1 \\
A \in \mathscr{A}}}\left|\left(\omega_{1}-\omega_{2}\right)(A)\right| \\
=\left\|\omega_{1}-\omega_{2}\right\| .
\end{aligned}
$$

Using Lemma 2

$$
\begin{aligned}
\left|s_{\Lambda}\left(\omega_{1}\right)-s_{\Lambda}\left(\omega_{2}\right)\right| & =\frac{1}{V(\Lambda)}\left|\sum_{k} f\left(\lambda_{k}^{1}\right)-f\left(\lambda_{k}^{2}\right)\right| \\
& \leqq \frac{1}{V(\Lambda)} \sum_{k}\left|f\left(\lambda_{k}^{1}\right)-f\left(\lambda_{k}^{2}\right)\right| \\
& \leqq \frac{1}{V(\Lambda)} \sum_{k} 2\left|\lambda_{k}^{1}-\lambda_{k}^{2}\right|+f\left(\left|\lambda_{k}^{1}-\lambda_{k}^{2}\right|\right) .
\end{aligned}
$$

Let $\varepsilon_{k}=\left|\lambda_{k}^{1}-\lambda_{k}^{2}\right|$, then

and by (1)

$$
\left|s_{\Lambda}\left(\omega_{1}\right)-s_{\Lambda}\left(\omega_{2}\right)\right| \leqq \frac{1}{V(\Lambda)} \sum_{k} 2 \varepsilon_{k}+f\left(\varepsilon_{k}\right)
$$

$$
\sum_{k} \varepsilon_{k}=a \leqq\left\|\omega_{1}-\omega_{2}\right\| .
$$

The case $a=0$ implies $\varepsilon_{k}=0$ for all $k$ and hence

$$
\left|s_{\Lambda}\left(\omega_{1}\right)-s_{\Lambda}\left(\omega_{2}\right)\right|=0 .
$$

If $a>0$ then by the convexity of the logarithm

$$
\begin{aligned}
& \frac{1}{V(\Lambda)} \sum_{k} 2 \varepsilon_{k}+f\left(\varepsilon_{k}\right)=\frac{2 a}{V(\Lambda)}+\frac{a}{V(\Lambda)} \sum_{\varepsilon_{k} \neq 0} \frac{\varepsilon_{k}}{a} \log \frac{1}{\varepsilon_{k}} \\
& \leqq \\
& \quad \frac{2 a}{V(\Lambda)}+\frac{a}{V(\Lambda)} \log \sum_{\varepsilon_{k} \neq 0} \frac{1}{a} \leqq \frac{2 a}{V(\Lambda)}+\frac{a}{V(\Lambda)} \log \left(\frac{N^{V(\Lambda)}}{a}\right) \\
& =\frac{2 a-a \log a}{V(\Lambda)}+a \log N .
\end{aligned}
$$

Hence

$$
\begin{aligned}
\left|s\left(\omega_{1}\right)-s\left(\omega_{2}\right)\right| & =\lim _{\Lambda \rightarrow \infty}\left|s_{\Lambda}\left(\omega_{1}\right)-s_{\Lambda}\left(\omega_{2}\right)\right| \\
& \leqq\left\|\omega_{1}-\omega_{2}\right\| \log N .
\end{aligned}
$$




\title{
References
}

1. Lanford, O., Robinson, D. W.: J. Math. Phys. 9, 1120 (1968).

2. Fannes, M.: The entropy density of quasi free states, Preprint July 1972 KUL Leuven Belgium.

3. Kato, T.: Perturbation theory for linear operators. Theorem III.6.10. Berlin-Heidelberg-New York: Springer 1966.

\author{
M. Fannes \\ Instituut voor theoretische fysica \\ Dept. Nat. Celestijnenlaan 200D \\ B-3030 Heverlee, Belgium
}

